\title{
内蒙古达类湖地区蒙原羚繁殖期及其前后昼间行为 时间分配及能量平衡策略
}

\author{
刘丙万 ${ }^{1, *}$ ，钱执强 ${ }^{1}$, 张 博 ${ }^{1}$, 刘松涛 ${ }^{2}$, 黎 明 $^{2}$ \\ (1. 东北林业大学 野生动物资源学院, 哈尔滨 150040; 2. 内蒙古达妻湖国家级自然保护区, 呼伦贝尔 021008)
}

摘要: 2007 年 11 月、 12 月和 2008 年 3 月, 在内蒙古达㐘湖地区, 采用扫描取样法对雌雄蒙原羚繁殖期及 其前后昼间行为时间分配进行了研究。研究表明: (1) 繁殖期前、繁殖期和繁殖期后, 雌性蒙原羚采食时间, 占昼间活动时间的比例分别为 $(44.9 \pm 3.8) \% 、(43.5 \pm 4.0) \%$ 和 $(46.2 \pm 3.1) \%$; 卧息时间，占昼间活动时间的 比例分别 为 $(32.3 \pm 4.8) \%$ 、 $(29.2 \pm 2.9) \%$ 和 $(28.0 \pm 4.8) \%$; 雌性蒙原羚在繁殖期及其前后采食、移动和卧息 的行为时间分配差异不显著 $(P>0.05)$, 站立、繁殖、 “其他” 行为时间分配差异性显著 $(P<0.05)$ 。(2) 繁殖期前、 繁殖期和繁殖期后，雄性蒙原羚采食时间，占昼间活动时间的比例分别为 $(52.6 \pm 3.8) \% 、(17.5 \pm 2.8) \%$ 和 $(29.8$ $\pm 4.8) \%$; 卧息时间，占昼间活动时间的比例分别为 $(13.4 \pm 6.4) \% 、(24.2 \pm 4.1) \%$ 和 $(44.2 \pm 4.7) \%$ 。雄性蒙原羚 在繁殖期及其前后采食、卧息、站立、移动、繁殖、“其他” 时间分配均有显著差异 $(P<0.05)$ 。动物采食卧息的 行为时间分配反映动物的能量平衡策略。雌性蒙原羚的时间分配表明, 雌性蒙原羚的能量平衡策略在繁殖期前、 繁殖期和繁殖期后没有发生显著变化, 均为能量摄入最优化策略, 尽可能多的时间分配在采食上; 雄性蒙原羚的 时间分配表明, 在繁殖期前, 其能量平衡策略为能量摄入最优化策略, 尽可能多的时间分配在采食上; 雄性蒙原 羚繁殖期及繁殖期后其能量平衡策略转变为能量支出优化策略, 尽可能少的支出能量, 尽可能多的时间分配在卧 息上。

关键词: 蒙原羚; 达㐘湖; 时间分配; 性别差异; 繁殖期

中图分类号: Q959.842; Q959.842.05 文献标识码: A 文章编号: 0254-5853-(2009)06-0694-05

\section{Diurnal Activity Budgets and Energy Balance Strategy of Mongolian Gazelle (Procapra gutturosa) Before, During and After the Rut Around Dalai Lake, Inner Mongolia}

\author{
LIU Bing-wan ${ }^{1, *}$, QIAN Zhi-qiang ${ }^{1}$, ZHANG Bo ${ }^{1}$, LIU Song-tao ${ }^{2}$, LI Ming ${ }^{2}$
}

(1. College of Wildlife Resources, Northeast Forestry University, Harbin 150040, China ; 2. Dalai Lake National Nature Reserve, Hulunbeier 021008, China)

\begin{abstract}
In November, December 2007 and March 2008, we studied the diurnal activity budgets of the Mongolian Gazelle (Procapra gutturosa) using group-scan-sampling for the time before, during and after the rut around DaLai Lake, Inner Mongolia. The activities of Mongolian Gazelle were divided into 6 categories: feeding, standing, moving, lying, rutting and others. The results showed: 1 ) Before the rut, the major behavior of the female was feeding, the time budget of feeding occupied $(44.9 \pm 3.8) \%$ of all diurnal time, the lying time was $(32.3 \pm 4.8) \%$; During the rut the females spent $(43.5 \pm 4.0) \%$ on feeding and $(29.2 \pm 2.9) \%$ on lying; After the rut the time budget of feeding occupied $(46.2 \pm 3.10)$ $\%$ of all diurnal time, the lying time was $(28.0 \pm 4.8) \%$. Activity budgets of the female on standing, rutting, others changed significantly among the three periods $(P<0.05)$, but activity budgets on feeding, moving, and lying did not change significantly among the three periods $(P>0.05) .2)$. Before the rut, the major behavior of the males was feeding, the time budget of feeding occupied (52.6 \pm 3.8$) \%$ of all diurnal time, the lying time was $(13.4 \pm 6.4) \%$; During the rut the males spent significantly less time on feeding $(17.5 \pm 2.8) \%$ and more time on lying $(24.2 \pm 4.1) \%$ compared with before the rut. After the rut, the time budget of feeding occupied $(29.8 \pm 4.8) \%$ of all diurnal time, while the lying time was (44.2 $\pm 4.7) \%$. Activity budgets of the male on feeding, standing, moving, lying, rutting, others changed significantly among the three periods $(P<0.05)$. The time budget on feeding and lying reflects on energy balance strategies. We conclude from
\end{abstract}


the results that; females do not change their strategy significantly among the three periods - they try to increase their energy intake; before the rut, the male is the same as the female, but during and after the rut, the males adopt an energy saving strategy to optimize their energy balance; males aim to save energy by spending more time lying.

Key words: Mongolian gazelle; Dalai Lake; Activity budgets; Sexual difference; Rut

动物通过行为适应其生活的环境。动物根据自 身的生理状况调整行为, 从而形成特定生理条件下 的时间分配规律(Flannigan，2002)。动物采食、卧 息行为时间分配反映该动物的能量平衡策略 (Christian \& Paul，2007)。研究动物行为时间分配, 对于揭示动物能量平衡策略有重要参考价值。动物 通常会采取采食时间尽可能长, 获得尽可能多能量 并维持收支平衡的能量摄入最优化的能量平衡策 略; 但当动物的能量获取无法满足其最低能量需求 时, 就有可能转变为能量支出最优化策略。卧息时 间分配尽可能多, 支出能量尽可能少 (Robbins, 1993)。

近年来, 蒙原羚行为学研究集中在集群行为、 迁徙行为和生境选择。Peter et al (2001)、Miura et al (2004) 和 Ito et al(2005)研究了蒙原羚的迁徙路线, 并将蒙原羚冬夏生境进行比较, 掌握了蒙原羚迁徙 与植被状况之间的关系和铁路对蒙原羚迁徙的影 响; Jin et al (2004)研究了蒙原羚的迁徙和集群行 为; Luo et al (2008)研究了影响达妻湖地区蒙原羚春 季生境选择的主要因素。为了验证雌雄蒙原羚在繁 殖期及其前后能量平衡策略是否发生了变化, 我们 在达赖湖地区研究了雌雄蒙原羚在繁殖期及其前 后的行为活动时间分配。

\section{1 研究地点和方法}

\section{1 研究地点}

研究主要在内蒙古达㐘湖地区（东经 $115^{\circ}$ $31^{\prime} \sim 117^{\circ} 43^{\prime}$, 北纬 $47^{\circ} 36^{\prime} \sim 49^{\circ} 50^{\prime}$ ) 完 成。该地区属于典型的中温带大陆性半干旱气候, 冬季寒冷漫长, 夏季温凉短促, 年平均气温 $-2 \sim 2$ ${ }^{\circ} \mathrm{C}$ 。无霜期短, 而积雪期长达 $140 \mathrm{~d}$ 左右, 年降雨 量 250 400 $\mathrm{mm}$, 主要集中于 7-8 月份 (呼伦湖 志 (续志一) 编撰委员会, 1998)。植被是我国温 带草原的一部分, 主要是针茅 (Stipa spp.) 草原、 羊草 (Agropyron cristantum) 草原和禾本-杂草草原。

\section{2 研究方法}

2007 年观察发现蒙原羚繁殖行为最早发生在 12 月初。因此, 我们把 2007 年 11 月定义为繁殖期
前, 2007 年 12 月定义为繁殖期, 2008 年 3 月定义 为繁殖期后。我们在 2007 年 11 月、 2007 年 12 月 和 2008 年 3 月, 利用扫描取样法对蒙原羚的昼间 行为时间分配进行了研究, 所有行为观测均由一人 完成, 行为观察时间包括整个昼间。由于草原上视 野开阔，同时蒙原羚的警惕性高，所以观察距离一 般为 $300 \sim 600 \mathrm{~m}$ ，使用高倍单筒望远镜（20-60 $\times 80 ）$ 进行观察。在研究中, 每隔 5 min 随机选取 蒙原羚个体。当一个群体中蒙原羚个数超过 10 只, 则随机选取 10 只; 不足 10 只蒙原羚, 选取全部个 体, 记录所发生的行为 (Jauhiainen \& Korhonen, 2005; Ryan et al, 2008)。动物个体行为持续时间不 超过 $5 \mathrm{~s}$ 时, 则认为行为没有发生变化。如蒙原羚 采食期间抬头观望，不超过 $5 \mathrm{~s}$ ，仍视为采食; 超过 $5 \mathrm{~s}$ 则视为站立。

根据对蒙原羚行为的预观察和有关研究结果, 本文将蒙原羚的行为分为 6 类 (Jiang, 2000; Liu \& Jiang, 2002; Lian et al, 2007):

采食（feeding）: 除饮水外, 站立或行走状态 下头部低于肩部水平线。

站立 (standing): 抬头观望，身体不发生位移。

移动（moving): 身体发生位移, 头部平行或 高于肩部水平。

卧息（lying)：以躺卧姿势伏于地面。

繁殖(rutting): 与繁殖相关的行为, 包括争斗、 追逐、爬跨等。

“其他” 行为(others)：除以上行为外的其他行 为, 包括修饰、排遗等。

去除人为干扰和记录时间少于 $30 \mathrm{~min}$ 的记录 后获得可用行为数据雌性蒙原羚共 $5460 \mathrm{~min}$ : 繁殖 期前 $1770 \mathrm{~min}$ (42 只次), 繁殖期 $1815 \mathrm{~min}$ (36 只次), 繁殖期后 $1875 \mathrm{~min}$ (47 只次); 雄性蒙原 羚共 $4955 \mathrm{~min}$ : 繁殖期前 $1650 \mathrm{~min}$ (23 只次), 繁 殖期 $1560 \mathrm{~min}$ (29 只次), 繁殖期后 $145 \mathrm{~min}$ (38 只次)。

\section{3 数据分析}

数据处理时将蒙原羚各行为活动时间转换为 该行为所用时间占昼间活动时间的比例。所有数据 
用 SPSS 11 进行统计分析, 数据以平均值士标准误 表示。在比较蒙原羚各行为在繁殖期及其前后所占 比例的差异性时采用单因素方差分析 (one-way ANOVA), 在比较各个时期雌雄蒙原羚两性行为差 异性时采用 $t$-检验。

\section{2 结 果}

\section{1 雌性蒙原羚行为时间分配}

雌性蒙原羚在繁殖期及其前后采食行为在昼 间活动时间中一直占较高的比例，在( $40 \% \sim 50) \%$

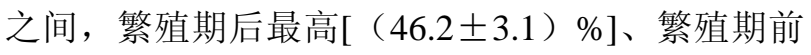

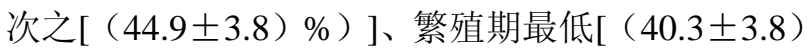
$\%$; 卧息行为次之, 在昼间活动时间中占 $30 \%$ 左 右, 繁殖期前最高[ $(32.3 \pm 4.8 ） \%]$ 、繁殖期次之

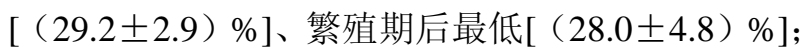
移动行为占 $10 \% \sim 15 \%$ 之间, 繁殖期最高[（14.3土 $1.7 ） \%] 、$ 繁殖期前次之[ $(13.1 \pm 2.8 ） \%]$ 、繁殖期 后最低 左右, 繁殖期后最高[ $(14.6 \pm 0.8) \%]$ 、繁殖期前 次之[ $(9.4 \pm 1.5) \%]$ 、繁殖期最低 $[(7.3 \pm 2.5) \%]$; 繁殖行为仅在繁殖期有记录, 在昼间活动时间中占 $(5.0 \pm 1.3) \%$; “其他” 行为最少, 在昼间活动时间 中均小于 1\%（图 1)。单因素方差分析结果表明, 雌性蒙原羚在繁殖期及其前后站立、繁殖、“其他” 行为活动时间占昼间活动时间的比例差异性显著 $(P<0.05)$; 雌性蒙原羚在繁殖期及其前后采食、 移动、卧息行为活动时间差异性不显著 $(P>0.05)$ 。

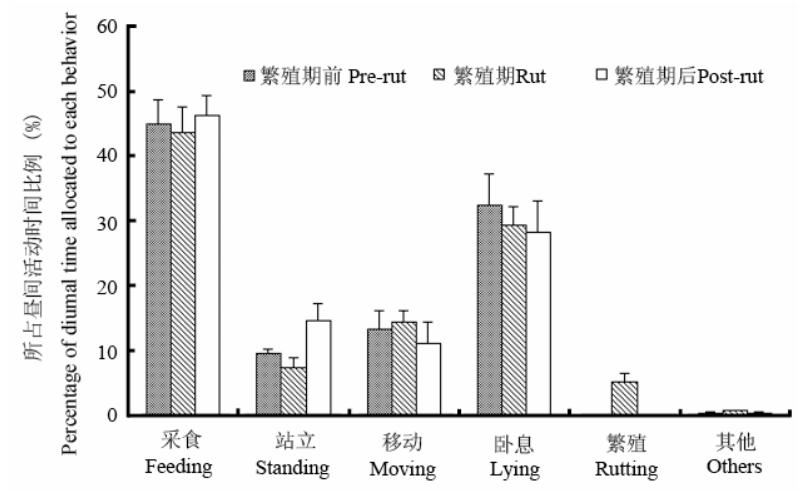

图 1 雌性蒙原羚繁殖期及其前后昼间行为时间分配

Fig. 1 Time budget of the female Mongolian Gazelle in different behaviors before, during and after the rut

\section{2 雄性蒙原羚行为时间分配}

雄性蒙原羚采食行为在繁殖期前, 占昼间活动
时间比例最高, 达(52.6土3.8)\%, 繁殖期最低, 占 昼间活动时间比例仅为 $(17.5 \pm 2.8) \%$ ，繁殖期后， 雄性蒙原羚采食的时间, 占昼间活动时间的比例 $(29.8 \pm 4.8) \%$; 繁殖期间站立行为和移动行为均高 于繁殖期前后，分别占 $(20.9 \pm 1.0) \%$ 和 $(29.4 \pm$ $2.2) \%$; 卧息行为占昼间活动时间比例逐渐增加, 繁 殖期前、繁殖期和繁殖期后依次为 $(13.4 \pm 6.4) \%$ 、

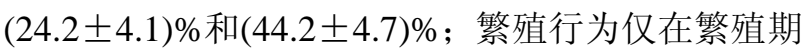

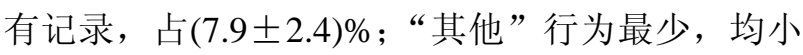
于 1\%（图 2)。单因素方差分析结果表明, 雄性蒙 原羚在繁殖期及其前后采食、站立、移动、卧息、 繁殖及 “其他” 行为活动时间占昼间活动时间的比 例均存在显著性差异 $(P<0.05)$ 。

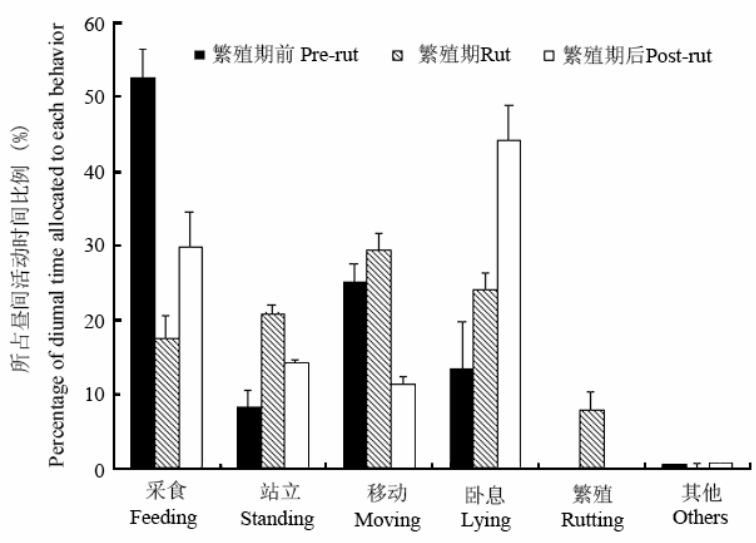

图 2 雄性蒙原羚繁殖期及其前后昼间行为时间分配

Fig. 2 Time budget of the male Mongolian Gazelle in different behaviors before, during and after the rut

\section{3 雌雄蒙原羚不同时期行为时间分配差异显著 性检验}

使用 $t$-检验对雌雄蒙原羚繁殖期及其前后行为 进行比较, 繁殖期前雌雄蒙原羚采食、站立和 “其 他” 行为活动时间占昼间活动时间的比例差异性不 显著 $(P>0.05)$, 移动和卧息的行为活动时间存在 显著差异 $(P<0.05)$; 繁殖期雌雄蒙原羚采食、站 立及移动活动时间占昼间活动时间的比例差异性 显著 $(P<0.05)$, 卧息、繁殖及 “其他” 行为活动 时间无显著差异 $(P>0.05)$; 繁殖期后雌雄蒙原羚 站立、移动、“其他” 行为活动时间占昼间活动时 间的比例无显著差异, 采食和卧息的行为活动时间 存在显著性差异 $(P<0.05)$ 。 


\section{3 讨 论}

\section{1 站立和移动行为时间分配}

在繁殖期前, 雄性蒙原羚的移动行为也占较大 比例，占昼间活动时间的(25.2土2.4) \%。这可能是 由于研究地区近年来蒙原羚的数量急剧减少, 分布 区域广，加之蒙原羚具有强的迁移性，所以面对即 将到来的繁殖期, 雄性蒙原羚可能为了增加遭遇雌 性的几率从而增加移动, 另外, 繁殖期前采食行为 较多, 占昼间活动时间的(52.6土3.8) \%, 在采食过 程中, 为了寻找食物必然伴随着移动行为的增加, 这可能也是繁殖期前移动行为较多的一个原因。动 物通过增加站立行为提供警戒性 ( Lima \& Bednekoff, 1999)。在繁殖期, 站立和移动行为对 于保证雄性蒙原羚的繁殖成功率有着重要的意义。 因此繁殖期雄性蒙原羚站立和移动时间增加, 共占 昼间活动时间的 50\%左右。动物频繁移动主要用于 巡逻和驱逐对手。我们观察到群主雄性蒙原羚为了 避免雌性蒙原羚和其他雄性蒙原羚的接触, 有时会 驱赶雌性蒙原羚使其远离其他雄性蒙原羚; 当某个 雌性个体离群较远时, 群主也会将其圈回群内, 随 着这种繁殖行为的发生, 常会伴随着大量移动行为 的发生。然而, 繁殖期雌性蒙原羚的移动行为虽较 繁殖期前后有所增加, 但并无显著差异, 可见繁殖 期并未对雌性蒙原羚的移动行为的时间分配产生 较大的影响。在繁殖期雄性蒙原羚担负了主要的警 戒任务, 这也使得雌性从中获益, 其警戒时间减少, 表现为站立活动时间仅为 $(7.3 \pm 1.5) \%$, 这一结果支 持了 Beauchamp（2003）的观点。

\section{2 采食和卧息时间分配及能量平衡策略}

在繁殖期前, 雄性野生动物对于配偶的竞争早 就已经开始 (Hardenberg et al, 2000)。在繁殖期前, 雄性蒙原羚为了储备能量, 采用了能量摄入最优化 策略, 采食行为占其昼间活动时间的 $50 \%$ 以上, Owen-Smith (1998) 在研究捻角羚 (Tragelaphus strepsiceros)时也发现了相似的结果。

\section{参考文献：}

Beauchamp G. 2003. Group size effects on vigilance: a search formechanisms [J]. Behav Processes, 63: 111-121.

Christian SW, Paul I. 2007. Feeding or Resting? The Strategy of Rutting Male Alpine Chamois [J]. Ethology, 113: 97-104.

Clutton-Brock TH, Green D, Hiraiwa-Hasegawa M, Albon SD. 1988. Passing the buck: resource defence, lek breeding and mate choice in
在繁殖期, 雄性蒙原羚减少了采食时间。在其 它一些物种的研究中, 也有过相似的结论。例如马 鹿 Cervus elaphus (Clutton Brock et al, 1982), 黄占 鹿 Cervus dama (Clutton-Brock et al, 1988), 驼鹿 Alces alces (Miquelle, 1990 ), 岩羚 Rupicapra rupicapra (Christian \& Paul, 2007) 的研究结果中 就显示，在繁殖期雄性采食时间显著下降，且卧息 的时间显著增多。甚至有研究显示雄性驼鹿在繁殖 期, 停止采食达 $18 \mathrm{~d}$ 之久 (Schwartz et al, 1984)。 有蹄类动物的采食行为节律受到环境因素（如食物 的质量和可获得性、大量积雪、低温等; Parker et al, 1996)、生理因素 (如肠道微生物群落的变化; Soest, 1994）等诸多因素的影响。繁殖期及其前后，雌性 蒙原羚采食和卧息的行为时间分配没有显著差异， 由此我们推测, 环境因素可能也不是雄性蒙原羚在 繁殖期采食时间减少的主要原因。

有蹄类的能量平衡策略主要随能量需求、食物 数量和质量的变化而变化的(Shi et al, 2003)。根据 能量平衡策略理论动物通常会采取能量摄入最优 化策略, 但当动物的能量摄入无法满足其最低能量 需求时, 其就有可转变为能量支出最优化策略

(Christian \& Paul, 2007)。研究表明雄性蒙原羚在 繁殖期及繁殖期后能量平衡策略转变为能量支出 最优化策略。由于一雄多雌的交配体制中雄性动物 以交配投资为主，为了提高自己的繁殖成功率，雄 性蒙原羚将更多的时间花在了站立、移动、繁殖行 为上，从而压迫了采食时间和卧息时间，但卧息的 时间仍有显著增加。在繁殖期后，随着繁殖及相关 行为的减少和消失，雄性蒙原羚将更多的时间分配 在采食和卧息的行为上。但雄性蒙原羚采食行为时 间占昼间活动时间的比例没有恢复到繁殖期前的 水平，仍有显著下降。卧息的时间增加迅速，卧息 行为成为雄性蒙原羚繁殖期后的最主要行为。

在繁殖期及其前后雌性蒙原羚始终采取的是 能量摄入最优化策略, 采食行为始终是雌性蒙原羚 的最主要行为。

fallow deer [J]. Behavl Ecol Sociobiol, 23: 281-296. Clutton-Brock TH, Guinness F, Albon SD. 1982. Red Deer. Behavior and Ecology of Two Sexes [M]. Chicago: University of Chicago Press.

Flannigan G, Stookey JM. 2002. Day-time budgets of pregnantmares housed in tie stalls: a comparison of draft versus lightmares[J]. Appl Anim Behav Sci, 78: 125-143. 
Hardenberg A, Bassano B, Peracino A, Lovari S. 2000. Male Alpine chamois occupy territories at hotspots before mating season [J]. Ethology, 106: 617-630.

Ito TY, Lhagvasuren N, Enkhbileg B, Takatsuki D, Tsunekawa S, Jiang ZW. 2005. Preliminary evidence of a barrier effect of a railroad on the migration of Mongolian Gazelles[J]. Conserv Biol, 19(3): 945-948.

Jauhiainen L, Korhonen HT. 2005. Optimal behaviour sampling and autocorrelation curve: modelling data of farmed foxes [J]. Acta Ethol, 8: 13-21.

Lima SL, Bednekoff PA. 1999. Back to the basics of antipredatory vigilance: can nonvigilant animals detect attack? [J]. Anim Behav, 58: 537-543.

Miura N, Ito TY, Lhagvasuren B, Enkhbileg D, Tsunekawa A, Takatsuki S, Jiang Z, Mochizuki K. 2004. Analysis of the seasonal migrations of Mongolian Gazelle, using modis data [J]. Int Arch Photogram Rem Sens Spat Inform Sci, 35(7): 418-422.

Miquelle DG. 1990. Why don' t bull moose eat during the rut[J]? Behav Ecol Sociobiol, 27: 145-151.

Owen-Smith N. 1998. How high ambient temperature affects the daily activity and foraging time of a subtropical ungulate, the greater kudu (Tragelaphus strepsiceros) [J]. J Zool : London, 246: 183-192.

Parker KL, Gillingham MP, Hanley A, Robbins CT. 1996. Foraging efficiency: energy expenditure versus energy gain in free-ranging black-tailed deer[J]. Can J Zool, 74: 442-450.

Peter L, William J, McShea, Christopher J, Brookes, Lhamsuren B, Chris W, Chris L. 2001. Spatial patterns in relative primary productivity and gazelle migration in the Eastern Steppes of Mongolia[J]. Biol Conserv, 102: 205-212.

Robbins, CT. 1993. Wildlife Feeding and Nutrition. 2nd ed [M]. San Diego: Academic Press, Inc.

Ryan R, Wilson, Brian D, Jansen, Paul R, Krausman. 2008. Planning and assessment of activity budget studies employing instantaneous
sampling[J]. Ethology, 114: 999-1005.

Schwartz CC, Regelin WL, Franzmann AW. 1984. Seasonal dynamics of food intake in moose[J]. Alces, 20: 223-244.

Shi JB, Dunber RI, Buckland D, Miller D. 2003. Daytime activity budgets of feral goats (Capra hircus) on the isle of rum: influence of season, age, and sex[J]. Can J Zool, 81: 803-815.

Soest PJ. 1994. Nutritional Ecology of the Ruminant [M]. 2nd ed. Ithaca: Cornell University Press.

Compiling committee of Chronicle of Hulun Lake. 1998. Chronicle of Hulun Lake [M]. Hohhot: Culture press of Inner-Mongolia. [《呼伦湖 志》 (续志一) 编撰委员会. 1998. 呼伦湖志（续志一）. 呼和浩特： 内蒙古文化出版社.]

Jiang ZG. 2000. Behavior coding and ethogram of the PÈRE David's deer[J]. Acta Theriol Sin, 20(1): 1-12. [蒋志刚. 2000. 麇鹿行为谱 PAE 编码系 统. 兽类学报, 20(1): 1-12.]

Jin K, Ma JZ, Gao ZX. 2004. Study of colony and migration of Mongolian Gazelles [J].Chn Herbiv, 24(4): 53-55. [金 崑, 马建章, 高中信. 2004. 黄羊的集群与迁徙. 中国草食动物, 24(4): 53-55.]

Lian XM, Zhang TZ, Cao YF, Cai ZY, Su JP. 2007. Diurnal behavioral time budgets and activity rhythm of the female Tibetan antelope (Pantholops hodgsoni) in summer[J]. Acta Theriol Sin, 27(1): 53-57. [连新明, 张同作, 曹伊凡, 蔡振媛, 苏建平. 2007. 夏季雌性藏羚昼 间行为时间分配及活动节律. 兽类学报, 27 (1): 53-57.]

Liu BW, Jiang ZG. 2002. Foraging strategy of Przewalskis Gazelle (Procapra Przewalskii) [J]. Acta Zool Sin, 48(3): 309-316. [刘丙万, 蒋志刚. 2002. 普氏原羚的采食对策. 动物学报, 48(3): 309-316.]

Luo ZH, Liu BW, Liu ST. 2008. Spring habitat selection of Mongolian Gazelle (Procapra gutturosa) around Dalai Lake,Inner-Mongolia [J]. Acta Theriol Sin, 28(4): 342-352. [罗振华, 刘丙万, 刘松涛. 2008. 内 蒙古达㐘湖地区蒙原羚的春季生境选择. 兽类学报, 28(4): 342-352.] 